\title{
Children and Pain: Letter to the Editor
}

\section{Ishan Poudel*}

Internal Medicine, California institute of Behavioral Neurosciences and Psychology, USA

*Corresponding Author: Ishan Poudel, Internal Medicine, California institute of Behavioral Neurosciences and Psychology, California, USA.

Received: August 30, 2019; Published: September 13, 2019

DOI: 10.31080/ASPE.2019.02.0149

I am incredibly delighted to read the Clinical Review "Children and Pain" published in ACTA Scientific Paediatrics on $8^{\text {th }}$ of August 2019 [1]. I am also grateful to the journal for publishing an article, that explores such an important aspect in clinical pediatrics which most of the modern-day practitioners either chose to ignore or have never explored.

The authors have tried to discuss an important aspect of clinical management of pain in infants and children who cannot verbalize their symptoms [1]. Most of the time, the clinical judgment combined with exhibited signs are taken into consideration for pain management in these patient population. However, we do not have an exact measure of how much and when to provide pain medication. We should appreciate the author's effort in bringing an issue of global importance that has been hidden for so long and open a new era of discussion in clinical Pediatrics. We should also admire the view of authors expressing treatment of pain to be the original right for every infant and children [1].

Finally, on behalf of like-minded clinicians, I would like to thank the editors and the journal itself for providing a platform for such a paramount clinical review.

\section{Conflict of Interest}

None.

\section{Bibliography}

1. Fatma Abdellah Mohamed. "Children and Pain". Acta Scientific Paediatrics 2.8 (2019): 30.

Volume 2 Issue 10 October 2019

(C) All rights are reserved by Ishan Poudel. 